\title{
GESTIÓN DE LA INFORMACIÓN Y LA RESPONSABILIDAD SOCIAL EMPRESARIAL
}

\section{INFORMATION MANAGEMENT AND CORPORATE SOCIAL RESPONSIBILITY}

Autor 1 - Víctor Daniel Bedoya García

Licenciado en inglés como Lengua Extranjera UNAD, Especialista en Educación Superior a Distancia UNAD, estudiante de Maestría en Administración de Organizaciones UNAD. Integrante del Semillero Innovación y Desarrollo I+D - GRINDES

Correo electrónico: vdbedoyag@unadvirtual.edu.co

ORCID: https://orcid.org/0000-0002-4266-4580

Autor 2 - Marleny Torres Zamudio

Economista, Especialista en Finanzas, Magister en Administración, candidata a Doctora en Ciencias de la Educación.

Investigadora grupo GRINDES. Docente Asociada, Universidad Nacional Abierta y a Distancia (UNAD), Tunja - Colombia.

Correo electrónico: marleny.torres@unad.edu.co

ORCID https://orcid.org/0000-0002-9091-5831

Google Académico:

https://scholar.google.com.co/citations?user=5bt7zwMAAAAJ\&hl=es

\section{RESUMEN.}

El objetivo de la investigación que da origen a este documento es determinar la incidencia de la gestión de la información en la toma de decisiones empresariales y en la generación de conocimiento al interior de las organizaciones, en el marco de la Responsabilidad Social Empresarial. Se realizó una investigación descriptiva, mediante un ejercicio de revisión documental, con la consideración de tres categorías de análisis: la gestión de la información organizacional, la gestión del conocimiento, y una categoría conjunta que analiza la Responsabilidad Social Empresarial (RSE) y gestión de la información. La discusión y resultados parciales que se presentan aquí describen el proceso de toma de decisiones fundados en la gestión de la información, dentro de ello, 
su incidencia en la toma de decisiones relativas a los aspectos económicos, sociales y ambientales en el marco de la RSE, que viabilizan la creación de círculos virtuosos y aportan al desarrollo competitivo de las organizaciones, al bienestar de las comunidades, y a la sustentabilidad ambiental.

\section{Palabras Clave.}

Conocimiento, información, Responsabilidad Social Empresarial, toma de decisiones, vigilancia tecnológica.

\section{ABSTRACT.}

The objective of the research that gives rise to this document is to determine the incidence of information management in business decision making and in knowledge management, within the framework of Corporate Social Responsibility. A descriptive research was conducted through a documentary review with the consideration of three main categories of analysis, organizational information management, knowledge management and a combined category that analyzes Corporate Social Responsibility, CSR and information management.

The discussion and partial results presented here describe the decisionmaking process based on information management and its impact on economic, social and environmental aspects within the framework of CSR, which enables the creation of virtuous circles and contributes to the competitive development of organizations, to the well-being of communities and to environmental sustainability.

\section{Keywords.}

Knowledge, information, Corporate Social Responsibility, decision making, technological vigilance

\section{INTRODUCCIÓN.}

\section{La información, el conocimiento y la toma de decisiones empresariales}

En el marco de sociedad de la información y del conocimiento, las organizaciones evidencian la importancia de gestionar asertivamente la información, pues, más que detentar inconmensurables cantidades de 
datos e información, su adecuada administración, procesamiento y análisis contribuyen en la toma de decisiones que les conducirán hacia la perdurabilidad, a la luz de su visión estratégica y la comprensión de su realidad interna y externa, así como de los inminentes cambios que les sobrevienen en la marcha.

En un momento en donde abunda la información, su administración, en plena era digital, demanda el uso de herramientas que posibiliten su gestión. El valor de la información, más allá de facilitar análisis, sugerencias, señalamientos y advertencias, se representa en la posibilidad de cimentar la creación de significados y conocimiento que dinamizan la productividad y fundamentan la gestión de decisiones asertivas o, en el peor de los casos, menos riesgosas. Así las cosas, en un entorno volátil, incierto y de cambios inopinados, la información y el conocimiento resultante se configuran como los instrumentos que posibilitan una preeminente continuidad operativa de las organizaciones.

En el ámbito de las organizaciones, la información puede ser gestionada en torno a múltiples intereses y pretensiones, aunque es un proceso subsecuente, pues, no se puede pensar en la creación de conocimiento, por ejemplo, si no se ha gestionado la información con base en modelos y estrategias. La gestión del conocimiento organizacional, inclusive la gestión de las decisiones, no pueden quedar al albur. Se necesita de información que permita dar a conocer la realidad endógena y exógena de las organizaciones, disminuir la incertidumbre, paliar el riesgo, crear conocimiento, innovaciones, productos o servicios en función de las necesidades y pretensiones.

Así las cosas, hoy en día, las organizaciones demandan el uso de instrumentos y herramientas articuladas mediante una base de conocimiento en donde se dé a conocer todo lo que acaece con los grupos de interés, pues, sean internos o externos, estos promueven la valorización del negocio mediante la participación e interacción. Por lo tanto, previo a emprender una solución asociada a la gestión del conocimiento, la implementación de una estrategia precursora de gestión de información resulta ser una acción indefectible (Gil et. al, 2011).

Para empezar, al hablar de información se torna fundamental distinguir el significado de dato, pues, como elemento independiente, corresponde generalmente a una representación algorítmica o alfanumérica atribuible a una variable cualitativa o cuantitativa según corresponda. En este sentido, en el contexto organizacional, los datos como tal resultan irrelevantes si no llevan implícitos un propósito (Carrión, 2017). 
Ahora, la información, en contraste con el dato, es aquella que tiene significado, propiamente, en función de los factores de relevancia y propósito alrededor de una temática, situación o realidad de interés. La trascendencia de la información a través de diferentes instrumentos relaciona emisores y receptores con una intencionalidad. Cuando se habla de flujo de mensajes, se asume la información y el flujo de esta se refiere a conocimiento (Nonaka y Byosiere, 2000, citado por Vega et. al, 2017).

Los datos, además de describir características independientes de las variables, son preparados para generar la información necesaria, convirtiéndose en un recurso estratégico necesario para la toma de decisiones (Bravo et. al, 2018). Por lo tanto, la información, que corresponde a un nivel superior, acude a la agrupación de datos que le dan cuerpo, vigor y significancia a un mensaje. Así se encauza la posibilidad de ejercer decisiones y de diseñar estrategias frente a las necesidades, prioridades y pretensiones de las organizaciones.

Se inicia, entonces, de los datos como elementos aislados y no orientativos, con una disposición de los mismos en sentido de un propósito (ver figura 1). Se establece la información que facilita los análisis, interpretaciones, juicios de valor y conocimiento, así como la creación de significados que soportan la toma decisiones, paralelamente, el diseño de planes y estrategias, en la visión de que las organizaciones se ven enfrentadas a grandes e inminentes retos (Moreno y Dueñas, 2018).

Figura 1

Proceso de gestión de la información a partir de datos

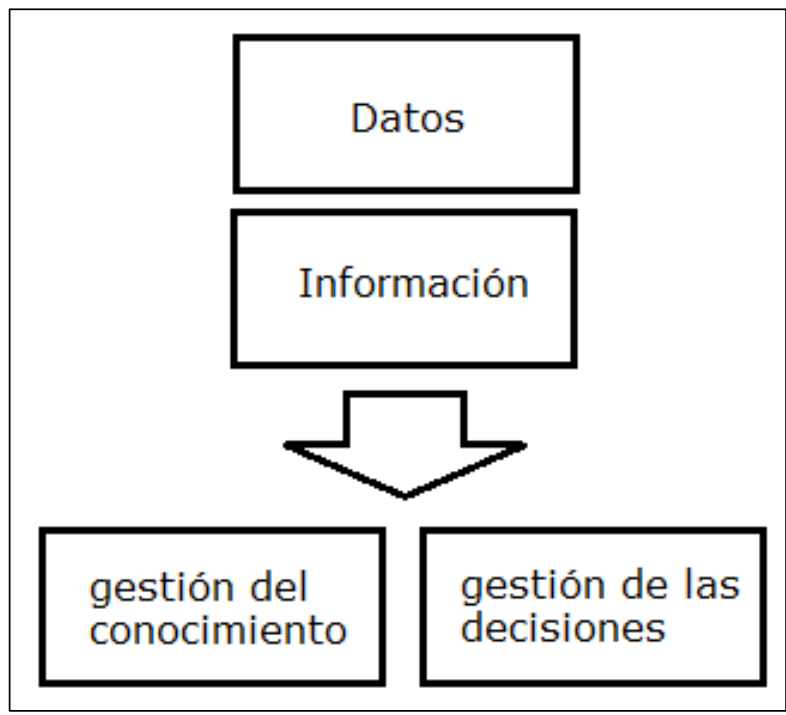

Fuente: elaboración propia 
Hoy, la articulación de las tecnologías digitales en la gestión organizacional está fuertemente marcada y apoyada por sistemas de información con ordenadores que acoplan cuatro elementos o componentes: personas, datos, procedimientos y tecnologías de información y comunicación (Lapiedra et. al, 2011).

Así las cosas, un sistema de información propicia entornos tanto de identificación e intercambio de información como de ideas para favorecer la generación nuevo conocimiento $y$, a la postre, de valor. Por tanto, el sistema debe permitir el uso de datos e información interna y externa, por lo que las organizaciones deben ser eficientes en términos de disponibilidad y acceso (Artiles y Márquez, 2013), comprendiendo que la operatividad de las organizaciones se ha re direccionado hacia nuevos focos de competitividad (Moreno y Dueñas, 2018).

Por tanto, se ha concebido la correcta gestión de la información como un imperativo, en orden de pretender la perdurabilidad y competitividad en el entorno de la nueva economía, además, el bienestar de todos en el marco de la RSE. Se torna sugerente para las organizaciones ejercer, estimular y catalizar el intercambio de información, saberes y significados al interior y entre las áreas de la empresa (Cornella, 1994, citado por Artiles y Márquez, 2013), sin desdeñase de la realidad y necesidades de los colaboradores externos, del entorno y el medio ambiente.

Ahora, como un acto tendencioso en las diferentes disciplinas, se habla indiscriminadamente de "modelo". A pesar que varios teóricos han cimentado otros constructos alrededor de este concepto, también se constituye una acepción sosegada para definir "modelo", inicialmente, como una teoría o paradigma, también, como un esquema o representación integrada por varios elementos o componentes en donde se describe una dinámica de funcionalidad mediante acciones o pasos a desarrollar. Un modelo, por tanto, puede ser la representación o ilustración gráfica de varias cosas, sea de una teoría, un proceso, procedimiento, sistema, mediante ayudas de carácter visual (Ponjuán, 2011).

Existen diferentes modelos que pretenden esquematizar variados procesos de gestión de información, y que pueden, a su vez, orientarse a diferentes propósitos como informar, advertir, sugerir o, a partir de la información generada, crear conocimiento y soportar la toma de decisiones. En este sentido, dichos modelos son diseñados o propuestos a la luz de diferentes objetivos y entornos, y caracterizan pasos, funciones, momentos y actores, así como su relacionamiento e interacciones a través de diferentes instrumentos y mecanismos. Como 
ya se expuso, un modelo puede ser la representación o ilustración gráfica de varias cosas, sea de una teoría, un proceso, procedimiento, sistema, mediante ayudas de carácter visual (Ponjuán, 2011).

Respecto a la gestión de la información, los modelos son intencionados a favorecer la creación de productos y servicios, los cuales, por su dinámica y comportamiento como sistemas abiertos, permiten transformar la información en la base para crear significados y conocimientos (Choo, 1996, citado por Gonzáles, 2014). Se pasa entonces de los datos a la información, en consideración de los sistemas y modelos de gestión por los cuales dicha información pueda ser canalizada y aprovechada, esta - la información - no corresponde a un recurso fungible.

En esta misma línea, la información se presenta como un insumo inagotable, multiplicable y transferible, y se configura como el asidero en donde estriba la creación de conocimiento. A partir de la asertiva gestión de la información, se pueden construir significados y crear conocimientos que permiten pertrechar la marcha de las organizaciones mediante una indicada gestión de decisiones y acciones, también, en función del beneficio no solo para las organizaciones, sino también para la sociedad y el medio ambiente en el marco de la RSE.

De esta manera, en el manuscrito se presentan los resultados parciales de un proceso investigativo que busca evocar la importancia de la gestión de la información en las organizaciones y su incidencia tanto en la toma de decisiones como en la creación de conocimiento en el marco de la RSE, es decir, en función del bienestar de quienes, incursos en su operatividad, hacen parte de las organizaciones, así como también de las comunidades y el medio ambiente.

\section{METODOLOGÍA.}

El desarrollo metodológico se realizó bajo un proceso secuencial de exploración, discriminación, selección y compilación de información para el establecimiento de análisis e interpretaciones alrededor de la temática central. Así las cosas, tanto el proceso heurístico como el hermenéutico fueron abordados mediante tres categorías de análisis: a) gestión de la información organizacional, b) gestión del conocimiento, c) una categoría conjunta que analiza la RSE y la gestión de la información.

Así, este estudio documental se presenta como preludio al diseño de un sistema de información empresarial que permita identificar necesidades de conocimiento de procesos clave de una organización en el marco de la RSE. Mediante un enfoque cualitativo de investigación, se ejerció una 
revisión bibliográfica en donde la información de interés fue clasificada y recolectada para recalar en la codificación, análisis e interpretación de la misma, y así lograr, subsecuentemente, la discusión y resultados parciales.

\section{DISCUSIÓN Y RESULTADOS PARCIALES.}

La dinámica contemporánea de la nueva economía suscita cambios de ritmo y dirección significativamente expeditos y abruptos, por ello, las organizaciones que no se aboquen a gestionar la información, a crear conocimiento y a gestionar sensata y acertadamente las decisiones pueden quedar eventualmente proscritas.

Es en este sentido que se corrobora la necesidad de diseñar estrategias, establecer procesos y ejercer acciones orientadas a gestionar la información, de modo que se logre catalizar el desarrollo holístico de las organizaciones. Por consiguiente, es importante gestionar inicialmente la información desde las acciones de captura y transferencia para proceder a la creación, usabilidad y aplicabilidad del conocimiento clave de cada proceso (Cardozo, 2018), que, a su vez, se presente como la plataforma en donde se soportan tanto la creación del conocimiento como la toma de decisiones.

Así, dentro de los procesos de carácter organizacional se ilustra la dinámica de cómo, enmarcados en un modelo de gestión de información, se articulan los datos para consolidar la información que da paso a la generación de conocimiento y la toma de decisiones, con la acción dirigida al posicionamiento estratégico, que estriba, como aludido previamente, en el aprovechamiento eficiente de la información (Rodríguez et. al, 2020).

Por tanto, para beneficio de la organización, es posible ejercer la vigilancia tecnológica para lograr dicho posicionamiento estratégico, pues, también, en el marco de la RSE y desde la misma gestión de la información, cabe establecer mayor proximidad y vínculo con la comunidad, mientras las organizaciones corresponden a todos sus colaboradores y a su objeto social aún en armonía con el medio ambiente (Ver figura 2).

Por lo anterior, en la necesidad de gestionar la información, se torna taxativo comprender que abordar la vigilancia tecnológica, apoyado en los avances de las tecnologías digitales del último tiempo (García y Torres, 2016), conlleva a las organizaciones a la captación, análisis e interpretación de la información para contribuir a la toma de decisiones (Rodríguez et. al, 2020), y a la creación de conocimiento, que, en la 
búsqueda de un mayor impacto, pueden enmarcase en la RSE, acudiendo a los compromisos endógenos, comunitarios y ambientales.

Figura 2

Encadenamiento gestión de la información y RSE

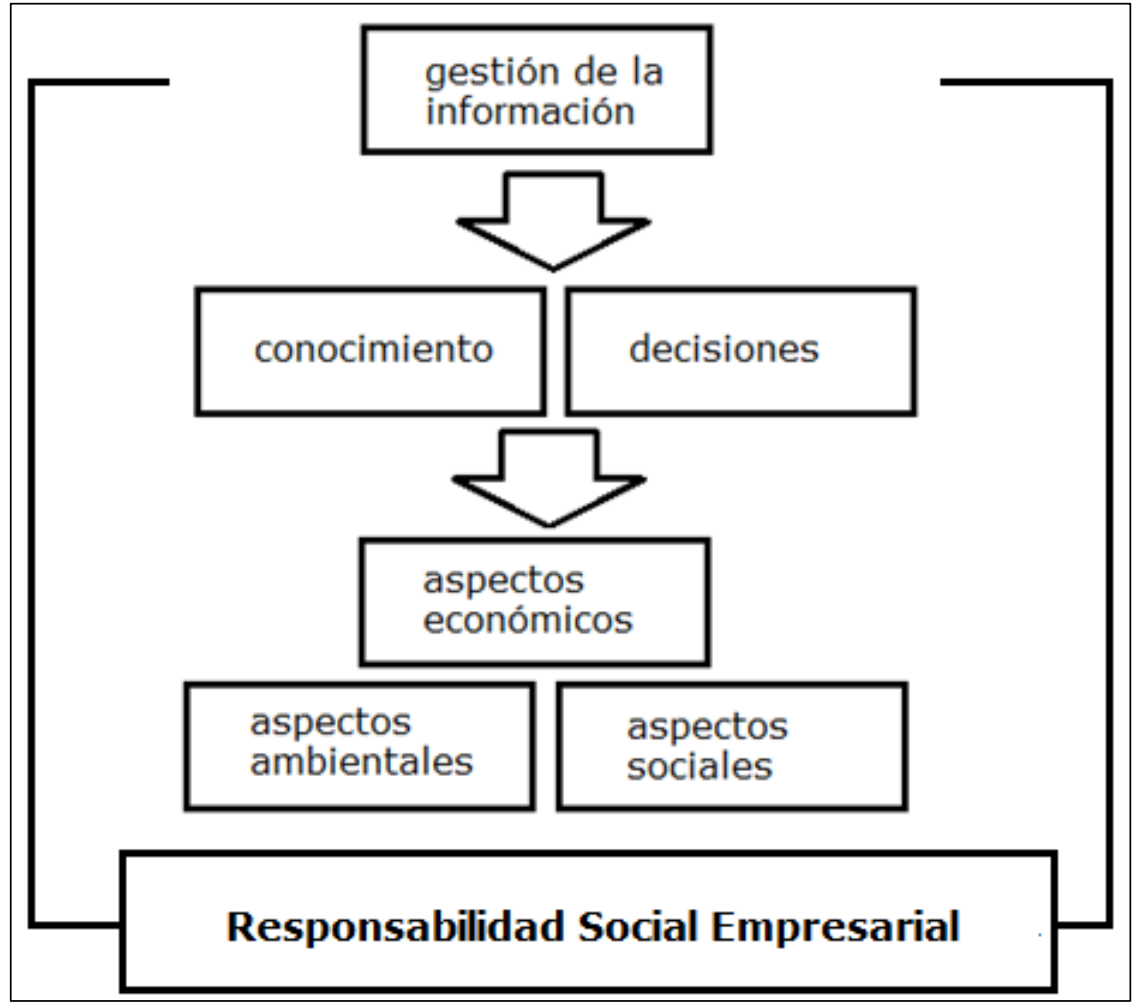

Fuente: elaboración propia

\section{RSE y Gestión de la información}

Aunque el mundo apela por repensar muchas actividades que sugieren un mayor interés por los aspectos no solo económicos, sino también sociales y ambientales, la RSE en muchos contextos parece ser todavía un apostolado sobre el verdadero "deber ser" de las organizaciones. Hace menos de una década se decía que las prácticas socialmente responsables no lograban mayor interés por parte del sector empresarial. La RSE se solía confundir con acciones filantrópicas, es decir, parecía más un favor de buena voluntad que contrastaba con el hecho internalizar la responsabilidad consustancial con todos los grupos de interés de las organizaciones, precisamente, en virtud del talento humano, de la sociedad, del medio ambiente (Valle, 2011).

La RSE es anuente con una variada letanía de acepciones y aproximaciones teóricas, por ello, en muchos entornos en donde dicha gestión todavía es incipiente, el tino de muchas organizaciones es, casi siempre, la única y la mejor forma concebida para su abordaje. Dichas 
acepciones y constructos recalan mayormente en la premisa de que la RSE obedece al cumplimiento al interior de la organización y su impacto externo en lo económico, social y ambiental (Rubio y Ferro, 2016).

Sin embargo, en la investigación adelantada por Lorenzoni (2020) para el caso colombiano, desde la mirada jurisprudencial, existen los fundamentos constitucionales para abordar la RSE, al amparo de la Constitución Política, en donde se establece la función social de la actividad empresarial y el principio de solidaridad, asumiendo, de esta manera, la responsabilidad que tienen las empresas, que supera la producción de riqueza con su función y responsabilidad social.

En el mismo sentido, Benavidez y Villanueva (2019) plantean que existen varios grupos de interés frente a la implementación de la RSE en las organizaciones, por tanto, a la luz de ofrecer una guía orientativa, el cumplimiento sugerido está descrito en los estándares más representativos internacionalmente, como puede ser el caso de: ISO 26000, SA 8000, ISO 18000, ISO 45001, Principios del Pacto Global y Objetivos de Desarrollo Sostenible, entre otros.

La RSE, por tanto, puede ser abordada con base en diferentes metodologías, estrategias y mecanismos, asimismo, es posible centrarse simultanea o subsecuentemente en diferentes propósitos y grupos de interés, de acuerdo con la prioridad, alcance y posibilidades para cada momento. Se reconoce así, el valor que se germina desde la información a la gestión del conocimiento y su transferencia en respuesta a ese llamado a buscar la equidad y el beneficio social (González, Manzano, Torres, 2018). Mediante la gestión de la información se torna posible distinguir los aspectos de interés en cada una de los actores y áreas relativas a los aspectos organizacionales endógenos y exógenos.

Esto ha permitido entrever que la RSE, además de contribuir al desarrollo competitivo de una organización, en su dimensión interna, por ejemplo, corresponde al fortalecimiento de un ambiente laboral sano y afable, ya que los mismos colaboradores han destacado diferentes beneficios otorgados por la organización en torno a su bienestar, que confluyen en su formación personal y profesional y se retribuyen, a su vez, en la organización (Benavidez \& Villanueva, 2019). Lo mismo se estima cuando se mira a los colaboradores externos, el entorno en donde cada organización opera, y el ambiente que funge, a su vez, como sustento para la operatividad y vida de todos los agentes y las generaciones venideras.

Las acciones que describen los procesos de la gestión de la información se forjan en la captura de datos, los cuales serán eventualmente procesados y transformados en la información que recalará en los 
usuarios incursos en un sistema. Tomando la información que suministra el sistema, se encauza la dirección, control y operatividad de las organizaciones que, entre otros aspectos, permite realimentar y canalizar la gestión del conocimiento y las decisiones.

Por ello, el conocimiento, las decisiones, asimismo, las estrategias y las acciones que se viabilizan desde la gestión de la información consienten, con una mirada dilatada en el contexto de la nueva economía, guarnecer las lides de las organizaciones, la sociedad y el medio ambiente. Allí radica La importancia de gestionar la información en las organizaciones y se corrobora parte de su ponderada incidencia en el marco de la RSE.

\section{CONCLUSIONES.}

En el ámbito organizacional, la gestión de la información y del conocimiento pueden orientar la toma de decisiones dirigidas allende del bienestar pretendido en un momento específico, pues, se viabiliza una utilidad y beneficio duradero para el talento humano, las organizaciones, el medio ambiente y la sociedad, en consciencia de las generaciones venideras.

Entre muchas otras alternativas de intervención y participación de las organizaciones en el marco de la RSE, será generalmente fructuoso considerar no solo el desarrollo competitivo y económico de las organizaciones, sino también el compromiso con los actores internos o externos, los territorios y el medio ambiente. Para ello, es importante partir de la gestión de la información en orden de identificar la realidad de cada dimensión - económica, social, ambiental -, de esta manera, priorizar y proceder en torno a los intereses y alcance.

\section{BIBLIOGRAFÍA.}

Artiles-Visbal, S. M., \& Márquez-Pérez, Y. (2013). El Modelo de Gestión de Información y Conocimiento: Resultados de su Aplicación en Una Empresa en Perfeccionamiento (Information and Knowledge Management Framework: Its Application Results in Company Upgrading). GECONTEC: Revista Internacional de Gestión del Conocimiento y la Tecnología, 1(1). Obtenido de: https://papers.ssrn.com/sol3/papers.cfm?abstract id=2396838

Benavides Parra, O. A., \& Villanueva García, Y. X. (2019). Responsabilidad social empresarial centrada en el bienestar de los colaboradores. Marco de realidad actual. Obtenido de:

https://repository.unad.edu.co/handle/10596/28560 
Bravo, C., Valdivieso, P. \& Arregui, R. (2018). Los sistemas de información en la toma de decisiones gerenciales en las empresas comerciales de Portoviejo. ECA Sinergia, 9(2), 45-54. Obtenido de: https://revistas.utm.edu.ec/index.php/ECASinergia/article/view/1334

Carrion, J. (2017). Diferencia entre dato información y conocimiento. Obtenido de:

http://148.202.167.116:8080/jspui/handle/123456789/869

del Valle, S. S. (2011). La Responsabilidad Social Empresarial: gestión estratégica para la supervivencia de las empresas. Dimensión empresarial, 9(2), 6-15. Obtenido de:

https://dialnet.unirioja.es/servlet/articulo?codigo $=3965840$

Devece Carañana, C. A., Lapiedra, R., \& Guiral Herrando, J. (2011). Introducción a la gestión de sistemas de información en la empresa. Universitat Jaume I. Obtenido de:

http://repositori.uji.es/xmlui/handle/10234/24161

García-Mogollón, A. M., \& Torres-Zamudio, M. (2017). Patent development in the peach production and transformation field: a technological monitoring study. Revista Ciencia y Agricultura, 14(1), 1529. Obtenido de:

https://dialnet.unirioja.es/servlet/articulo?codigo $=5966733$

Gil-Montelongo, M. D., López-Orozco, G., Molina-García, C., \& Bolio-Yris, C. A. (2011). La gestión de la información como base de una iniciativa de gestión del conocimiento. Ingeniería Industrial, 32(3), 231-237. Obtenido de: https://www.redalyc.org/pdf/3604/360433577009.pdfv

González, Y., Manzano, O., Torres, M. (2018). Categorías de análisis para un plan de responsabilidad social universitaria. ISBN: 978-958651-655-6, 1.a. ed. Bogotá: Sello Editorial UNAD.

https://doi.org/10.22490/9789586516556

González-Valiente, C. L. (2014). Midiendo la calidad de la información gestionada: algunas reflexiones conceptuales-

metodológicas. arXivpreprint arXiv:1406.6277. Obtenido de:

https://arxiv.org/abs/1406.6277

ISO. (2010). ISO 26000:2010 - Guía de responsabilidad social. Online Browsing Plaform (OBP). Obtenido de:

https://www.iso.org/obp/ui\#iso:std:iso:26000:ed-1:v1:es

Lorenzoni, L. (2020). Responsabilidad social empresarial en la jurisprudencia de la Corte Constitucional colombiana: dimensiones de obligatoriedad en la voluntariedad. Revista de Estudios Socio-Jurídicos, 
23(1), 1-23. [Publicación electrónica previa a la impresión] https://doi.org/10.12804/revistas.urosario.edu.co/ sociojuridicos/a.9085

Moreno-Cevallos, J. R., \& Dueñas-Holguín, B. L. (2018). Sistemas de información empresarial: la información como recurso estratégico. Dominio de las Ciencias, 4(1), 141-154. Obtenido de: https://www.dominiodelasciencias.com/ojs/index.php/es/article/view/72 $\underline{8}$

Orozco, Y. V. D., Acevedo, M. C., \& Acevedo, J. A. R. (2013). Responsabilidad Social Empresarial: Teorías, índices, estándares y certificaciones. Cuadernos de administración, 29(50), 196-206.

Obtenido de: https://www.redalyc.org/pdf/2250/225029797009.pdf

Ponjuán-Dante, G. (2011). La gestión de información y sus modelos representativos. Valoraciones. Ciencias de la Información, 42(2), 11-17. Objetivo: https://www.redalyc.org/pdf/1814/181422294003.pdf

Rubio, G. \& Fierro, F. (2016). La RSE: una teoría, diferentes visiones aplicadas. Administración \& Desarrollo, 46(2), 160-174. Obtenido de: https://dialnet.unirioja.es/descarga/articulo/6403474.pdf

Tejada, A. H. R., Betancur, A. M., Hidalgo, Á. M. M., \& Bohorquez, R. E. Á. (2020). Herramientas de simulación para la toma de decisiones empresariales. Documentos de Trabajo ECACEN, (2), 1-10. Obtenido de: https://hemeroteca.unad.edu.co/index.php/working/article/view/4262

Vega-Pérez, C. A., Grajales-Lombana, H. A., \& Montoya Restrepo, L. A. (2017). Sistemas de información: definiciones, usos y limitantes al caso de la producción ovina colombiana. Orinoquia, 21(1), 64-72. Obtenido de: http://www.scielo.org.co/pdf/rori/v21n1/0121-3709-rori-21-0100064.pdf 